Estudios Geológicos, 65(2)

julio-diciembre 2009, 157-165

ISSN: 0367-0449

doi:10.3989/egeol.39864.065

\title{
Etude de la structure du bassin d'Ouarzazate par sismique réflexion: Implications hydrogéologiques
}

\section{Study of the Ouarzazate basin structure by seismic reflection: hydrogeological implications}

\begin{abstract}
Kh. Boummane ${ }^{1}$, M. Jaffal ${ }^{2}$, A. Kchikach ${ }^{2}$
RÉSUMÉ

Les campagnes d'exploration pétrolière menées dans le bassin d'Ouarzazate ont permis l'acquisition d'une importante base de données de sismique réflexion. La présente étude concerne l'interprétation d'une partie de ces données dans l'objectif de caractériser la tectonique de l'Eocène et la structure de son système aquifère. Dans un premier temps, le réflecteur correspondant à la base de cet étage, représenté de grès et de calcaires, a été identifié, puis numérisé sur les différentes sections sismiques migrées-temps; ce qui nous a permis d'en établir une carte d'isochrones. Celle-ci montre que le secteur étudié est subdivisé en deux domaines très contrastés d'un point de vue structural. Le premier, septentrional, est affecté par une structuration intense de direction atlasique, tandis que le second, méridional, est faiblement plissé. Les résultats de la présente étude permettent une meilleure connaissance de la structure profonde du bassin d'Ouarzazate. Ceci permet de mieux appréhender le fonctionnement du système aquifère éocène et de rationaliser les futures campagnes de reconnaissance des eaux souterraines susceptibles d'être menées dans le bassin d'Ouarzazate.
\end{abstract}

Mots-clés: sismique réflexion, isochrone, Eocène, structure du bassin, hydrogéologie, bassin d'Ouarzazate Maroc.

\begin{abstract}
A large number of seismic reflection lines have been carried out in the Ouarzazate basin by the oil industry. The present study is concerned with the interpretation of a part of these data in order to characterize the structure of the Eocene aquifer system. The reflector corresponding to the base of this system, made up of sandstone and limestone, was first identified then digitized on each time-migrated seismic section. An isochrone map of this reflector was realized. The analysis of this map shows that the area under study is subdivided into two structurally contrasted domains. The first, the northern one, is intensively deformed; while the second, the southern one, is slightly folded. The results of this study provide a better understanding of the deep geological structure of the Ouarzazate basin. This allows us to better comprehend the functioning of the Eocene aquifer system, and to rationalize the future potential underground water exploration in the Ouarzazate basin.
\end{abstract}

Key words: seismic reflection, isochron, Eocene, basin structure, hydrogeology, Ouarzazate basin, Morocco.

\section{Introduction}

Le bassin d'Ouarzazate constitue avec ceux de Souss et d'Er-Rachidia-Boudnib-Erfoud une bande étroite qui s'étend en direction ENE-WSW entre le
Haut-Atlas et l'Anti-Atlas, plus connue sous le nom de «sillon sud-atlasique". Ce bassin se situe entre la chaîne du Haut-Atlas et les massifs anti-atlasiques du Saghrou et de l'Ougnate qui en constituent les principaux pourvoyeurs en matériaux de sédimenta-

\footnotetext{
${ }^{1}$ Laboratoire de GEOHYD, Département de Géologie, Faculté des Sciences Semlalia. Avenue My Abdellah, BP. 2390, Marrakech, Maroc. Email: khboummane@ucam.ac.ma

2 Laboratoire de Géoressources, Département des Sciences de la Terre, Faculté des Sciences et Techniques, BP. 549, Marrakech, Maroc. Email: jaffal@fstg-marrakech.ac.ma
} 


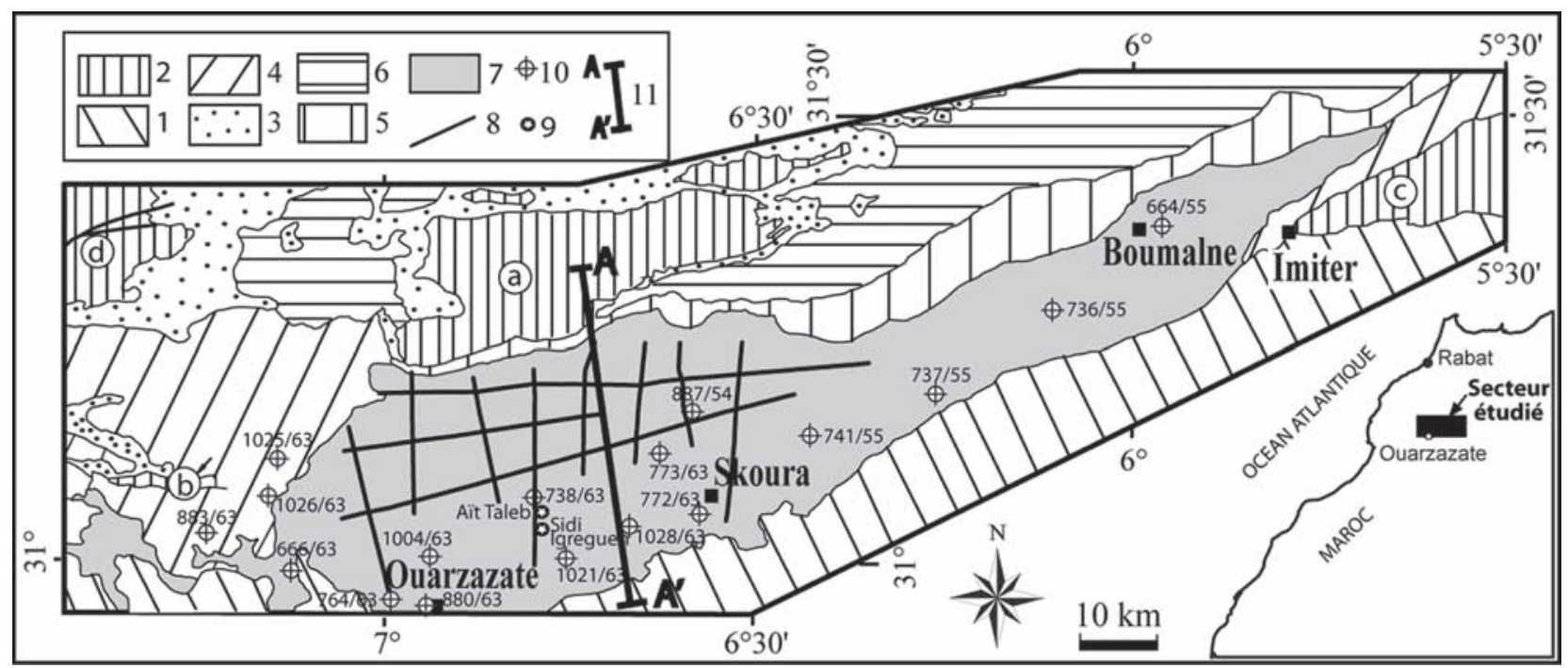

Fig. 1.-Situation de la zone d'étude sur la carte géologique schématique du pourtour du bassin d'Ouarzazate. 1. Précambrien de Jbel Saghro. 2. Boutonnières paléozoïques [a) Skoura, b) Imini, c) Imiter, d) Tichka]. 3. Trias et localement Lias externe. 4. Crétacé et Paléogène tabulaires. 5. Crétacé et Paléogène de la Zone sub-atlasique. 6. Domaine des nappes liasiques. 7. Néogène du bassin d’Ouarzazate. 8. Profil sismique. 9. Forage pétrolier. 10. Forage hydrogéologique. 11. Coupe de la figure 2.

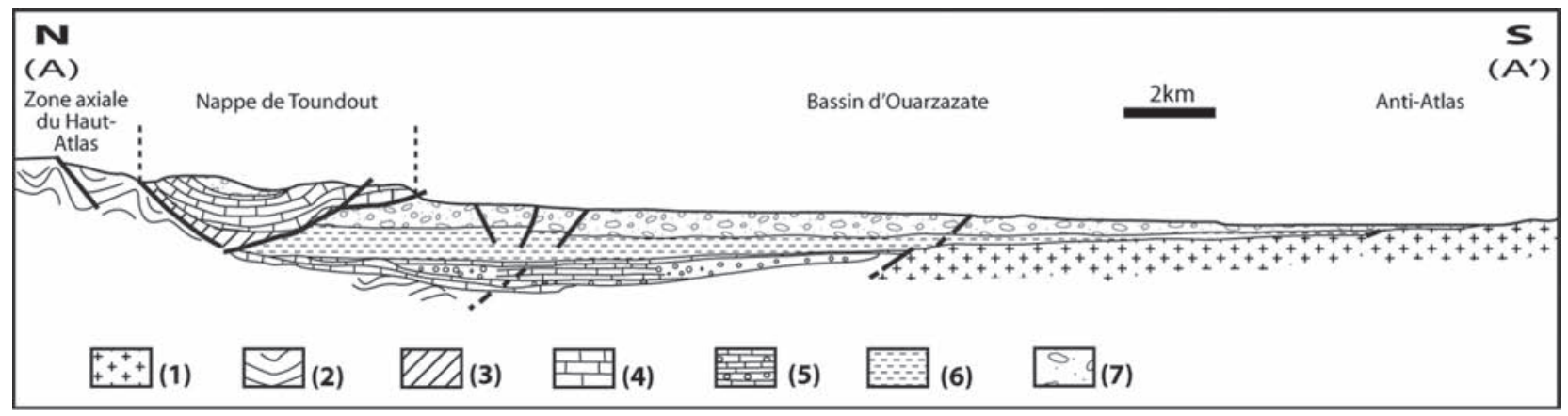

Fig. 2.-Coupe géologique à travers le bassin d'Ouarzazate (d'après Jossen \& Filali Moutei 1988, la coupe est localisée sur la figure 1). 1. Précambrien: roches magmatiques. 2. Paléozoïque: schistes, grès et quartzites. 3 . Trias: grès, pélites et basaltes. 4 . Lias inférieur et moyen: calcaires et dolomies. 5. Crétacé: conglomérats, sables argileux et barre calcaire du Cénomano-Turonien. 6 . Eocène: calcaires de l'Eocène inférieur, marnes grès rouges et conglomérats. 7. Mio-Pliocène: sables argileux et conglomératiques, conglomérats.

tion pendant le Cénozoïque (fig. 1). Il est limité à l'Ouest par le massif de Siroua et au Nord-est par le seuil d'Imiter qui le sépare du bassin d'Errachidia. Le bassin d'Ouarzazate forme une cuvette relativement étroite allongée en direction ENE-WSW sur une longueur de $160 \mathrm{~km}$ environ et dont la largeur maximum atteint $45 \mathrm{~km}$ aux méridiens d'Ouarzazate et de Skoura. Elle se rétrécit progressivement en allant vers le Nord-est où sa largeur n'est plus que de $20 \mathrm{~km}$ à Boumalne et $10 \mathrm{~km}$ à Imiter. Le centre de la cuvette est comblé par les dépôts néogènes et quaternaires (fig. 2). Il forme une surface plane, entaillée par des oueds et dont l'altitude moyenne varie entre 1100 et 1500 mètres (Jossen \& Filali Moutei, 1988; Sébrier et al., 2006).
Ce bassin fait partie d'une région où règne un climat aride à semi-aride, caractérisé par des précipitations très faibles (moins de 200 mm/an) et irrégulières, des écarts de température importants et une forte évaporation (2800 mm/an en moyenne) (Agoussine et al. 2004). Dans ces conditions climatiques défavorables, les eaux souterraines sont soumises à une exploitation intense, afin de subvenir aux besoins de plus en plus accrus de l'irrigation et de l'usage domestique de la population. Lors des deux dernières décennies, la succession de plusieurs années de sécheresse a exacerbé la situation en conduisant à un accroissement de l'activité de pompage des eaux souterraines et à une diminution des apports de leur renouvellement. Ceci a 
provoqué des modifications de l'état de la nappe soulignées notamment par une baisse du niveau piézométrique et une chute de la productivité des ouvrages. Les eaux souterraines sont devenues ainsi de plus en plus rares et certains forages de reconnaissance de ces eaux se sont révélés négatifs, à cause de l'insuffisance des connaissances de l'hydrogéologie régionale. Dans ces conditions, une intensification des investigations sur l'ensemble du bassin, notamment par les méthodes indirectes telles que les techniques géophysiques, devient nécessaire, en vue de définir les zones productives non encore identifiées.

C'est dans ce contexte que nous avons entrepris la présente étude, basée sur la compilation et l'interprétation des données sismiques enregistrées au cours des campagnes de prospection pétrolière menées dans le bassin d'Ouarzazate. Son principal objectif est de déterminer la structure profonde de ce bassin et plus particulièrement celle du système aquifère éocène. La base de ce système, qui constitue un bon réflecteur sismique, a été choisie comme niveau repère pour établir une carte $d$ 'isochrone que l'on propose d'analyser et de discuter.

\section{Contexte géologique et hydrogéologique}

Le bassin d'Ouarzazate est dominé par un recouvrement détritique continental mio-plio-quaternaire qui masque l'ensemble des terrains plus anciens à l'exception des dépôts crétacés et paléocènes qui affleurent à ses extrémités orientale et occidentale. Ce bassin repose au Sud sur les terrains précambriens de Jbel Saghrou (Görler et al. 1988), tandis qu'il limite au Nord la zone sub-atlasique. Cette zone est une bande étroite constituée de terrains crétacés et paléocènes, déformée par la tectonique atlasique et chevauchée par des nappes carbonatées du Lias. Le remplissage sédimentaire du bassin d'Ouarzazate est principalement constitué d'une série méso-cénozoïque reposant en discordance angulaire sur le socle paléozoïque qui affleure localement à la faveur des boutonnières de Tichka, Siroua, Imini et Errachidia. Cette série est formée de plusieurs unités lithostratigraphiques (fig. 3) (Costagliola \& Belkhir, 1992). Elle commence à la base par des dépôts triasiques rouges argilo-détritiques continentaux caractéristiques du Maroc. Cette première formation de nature continentale voire lagunaire est surmontée au Jurassique inférieur et moyen par une formation carbonatée, puis

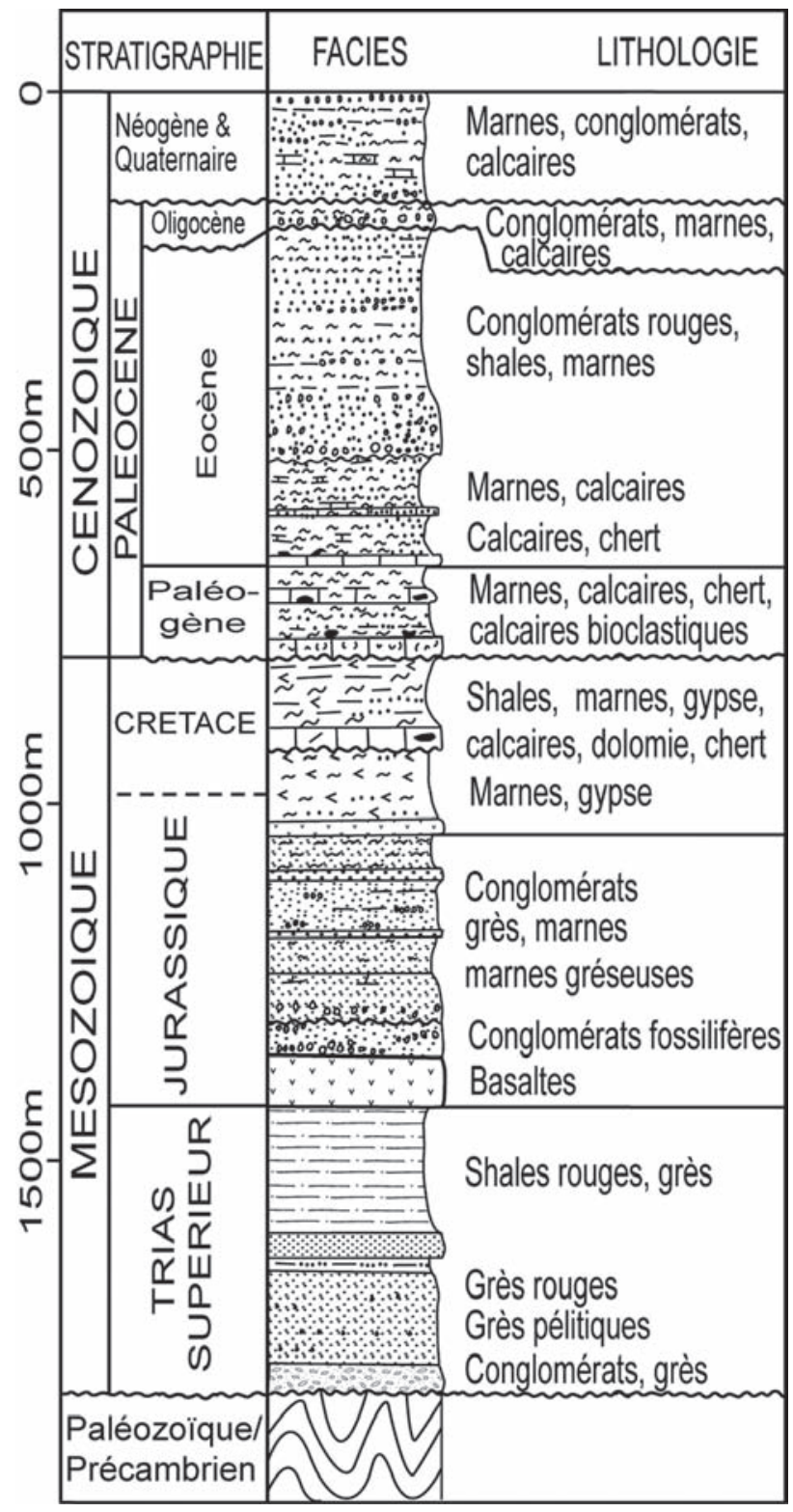

Fig. 3.-Log stratigraphique synthétique des formations mésocénozoïques du bassin d'Ouarzazate (Document ONHYM).

par une unité détritique gréso-conglomératique et argileuse passant vers le sommet à des argilites rouges gypsifères attribuée au Jurassique moyenCrétacé inférieur. Le Crétacé supérieur, reposant en légère discordance angulaire sur les termes précédents, est formé par une série carbonatée fossilifère, localement à silex à la base et par des dépôts argilogypsifères à passées de sel au sommet. Le Paléocène et l'Eocène inférieur et moyen sont représentés par des dépôts marins carbonatés, marneux et gréseux, localement phosphatés. Cet ensemble de près 
de $300 \mathrm{~m}$ de puissance est recouvert en légère discordance par des dépôts continentaux gréso-conglomératiques à lagunaires essentiellement détritiques localement gypsifères attribués à l'Eocène supérieur. Ce dernier se démarque du Crétacé supérieur par l'absence des dépôts marins et par l'existence de calcaires lacustres et de conglomérats abondants à proximité du Haut Atlas. Les faciès sont très conglomératiques dans la vallée du Dadès d'une épaisseur maximale de $400 \mathrm{~m}$.

L'absence de l'Oligocène dans l'ensemble de la zone est à relier à la phase principale de structuration du Haut-Atlas. Le Néogène et le Quaternaire sont constitués de dépôts continentaux conglomératiques, gréseux et argileux qui forment le recouvrement du bassin d'Ouarzazate. Cet ensemble est discordant sur tous les termes précédents.

D'un point de vue structural, le bassin d'Ouarzazate est caractérisé par la succession de deux phases tectoniques majeures: l'extension triasicojurassique et la compression atlasique (Oligocène). Ainsi, à la faveur d'une période distensive initiée dès le Trias et se poursuivant durant le Lias, va s'individualiser et se développer, le long de la bordure nord de l'Anti-Atlas, une zone subsidente qui correspondrait à la fosse ou rift atlasique. Son évolution tectonique, liée à des mouvements de bloc et des basculements, va être à l'origine de plusieurs discordances observables dans la succession mésozoïque. Les plus marquantes sont celles du sommet de la série continentale à lagunaire rouge du Trias supérieur et du Lias inférieur. Par la suite durant le Crétacé et le Paléogène, la zone reste relativement calme. La phase compressive atlasique d'âge oligocène, est responsable de la structuration de la chaîne de l'Atlas, autrement dit de l'inversion de la fosse atlasique (Laville et al., 1977). Cette inversion est en relation avec la convergence entre l'Afrique et L'Europe entamée au Cénozoïque et qui se continue actuellement (Laville et al., 1977; Dutour \& Ferrandini, 1985; Görler \& al., 1988; Fraissinet et al., 1988; El Harfi et al., 1996; Frizon de Lamotte et al. 2000, Morel et al., 1993, Morel et al., 2000; Amrhar, 2002). Les déformations liées à cette phase tectonique sont principalement observées dans la partie septentrionale du bassin, en particulier au niveau de la zone sub-atlasique et de la zone des nappes liasiques. Dans les zones méridionales, les terrains du Mésozoïque et du Cénozoïque se présentent sous forme d'un monoclinal à léger pendage nord, caractérisé par un amincissement progressif des couches en allant vers le Sud, sous l'action de diverses discordances (fig. 2). Cette évolution vers le Sud est liée à la présence au niveau de l'AntiAtlas de zones émergées qui serviront d'aires d'apport pour les sédiments détritiques.

D'un point de vue hydrogéologique, le bassin d'Ouarzazate abrite deux systèmes aquifères potentiellement intéressants pour l'exploitation des ressources hydriques souterraines de part leur accessibilité et leur potentiel volumique (Agoussine et al., 2004), ce sont :

- Les nappes profondes et semi-profondes correspondant aux calcaires du Lias, du Dogger et du Cénomano-Turonien; ainsi que les formations marines représentées par des grès coquilliers et des niveaux calcaires, séparés par des horizons marneux-carbonatés et des formations détritiques grossières, d'âge Paléocène et Eocène. Toutes ces formations sont susceptibles de constituer des réservoirs aquifères et constituent par conséquent des objectifs importants à reconnaître. D'où l'intérêt porté à l'aquifère Eocène dans cette étude.

- La nappe phréatique des formations quaternaires et mio-pliocènes, constituée, en réalité, d'un ensemble de nappes alluviales qui ont pour exutoire aval, l'oued Draâ et ses affluents.

\section{Données et méthodes}

Les données utilisées dans la présente étude proviennent de deux campagnes sismiques différentes réalisées dans le bassin d'Ouarzazate en 1980 et 1989 par la Compagnie Générale de Géophysique (CGG) dans le cadre d'un programme national de prospection pétrolière. Elles ont été acquises selon 11 profils formant une grille de 8 lignes transversales et 3 lignes longitudinales, permettant une bonne couverture du bassin. Les coupes sismiques sont disponibles à l'échelle 1/20000 sous forme de sections analogiques migrées-temps. Elles ont été enregistrées en couverture multiple d'ordre 60. Ces données ont subi les principaux traitements suivants, effectués par la CGG: démultiplexage au pas de $2 \mathrm{~ms}$, échantillonnage au pas de $4 \mathrm{~ms}$, édition des traces suivi d'un pré-filtrage dans la gamme de fréquences $12-70 \mathrm{~Hz}$, récupération des amplitudes, collection de points miroirs communs, déconvolution, correction statique des élévations topographiques des géophones au niveau moyen du sol (origine des temps), analyse continue de vitesses, correction dynamique, sommation en couverture 60 , 
correction statique du niveau moyen du sol au niveau de référence ou "datum plane", migration.

Le calage des données sismiques a été effectué en se basant sur les logs stratigraphiques des forages disponibles d'une part, et sur les données de la géologie de surface d'autre part. Les renseignements fournis par ces forages nous ont permis de localiser avec précision le réflecteur matérialisant le niveau repère que nous avons choisi pour étudier la structure du système aquifère éocène. L'attribution de ce réflecteur à l'Eocène est validée par les données géologiques de surface grâce à son affleurement vers l'Ouest. L'excellente qualité des sections sismiques a facilité la corrélation latérale de ce réflecteur entre les différents profils.

Les structures identifiées sur chaque coupe ont été reportées sur le plan de position des profils sismiques puis corrélées latéralement. Par la suite, le réflecteur correspondant à la base de l'éocène a été repéré puis numérisé sur les différents profils. Les profondeurs en temps-double obtenues pour ce horizon ont été interpolées à travers le réseau de lignes sismiques, à l'aide du logiciel GMT «Generic Mapping Tools" (Wessel \& Smith, 1995) en utilisant la méthode d'interpolation «continuous curvature splines in tension". Cette technique est préconisée quand on ne dispose pas d'une grande densité de données, comme c'est le cas de la présente étude, contrairement à d'autres telle que «near neighbor».

Il est évident que la densité des données ainsi que le choix de la méthode d'interpolation jouent un rôle primordial dans la fiabilité du résultat obtenu. Dans le cas de cette étude, même si on dispose d'une assez bonne couverture sismique du bassin d'Ouarzazate, on doit analyser avec précaution les structures mises en évidence par interpolation et ne considérer que celles qui sont contrôlées par les profils sismiques.

On a choisi la base de l'Eocène comme niveau repère, car le contact lithologique entre les calcaires et grès de l'Éocène (Cappetta et al. 1987) et la puissante formation marneuse du Paléocène est marqué par un changement brutal de faciès qui produit un fort réflecteur. Les renseignements fournis par les forages, ainsi que les données géologiques de surface grâce à son affleurement vers l'Ouest, nous ont permis de repérer et pointer avec précision ce réflecteur sur les différents profils. L'excellente qualité des sections sismiques a facilité sa corrélation latérale entre les différents profils et l'élaboration d'une carte d'isochrone de cet horizon.
L'intérêt que nous avons porté à l'Éocène dans le cadre de cette étude, est également justifié par le fait que celui-ci est reconnu comme étant l'un des plus importants aquifères du bassin d'Ouarzazate, et surtout qu'il est le moins profond après la nappe phréatique (Agoussine et al. 2004)

Signalons enfin que la présente démarche méthodologique a déjà été appliquée avec succès, sur des données similaires, dans un autre contexte de bassin sédimentaire marocain, en l'occurrence celui d'Essaouira (Jaffal et al. 2002).

\section{Discussion des résultats}

L'analyse détaillée d'une importante base de données sismique totalisant quelques $310 \mathrm{~km}$, a permis de caractériser la structure profonde du bassin d'Ouarzazate. La carte d'isochrones de la base de l'Eocène établie à l'issue de cette étude est présentée sur la figure 4. Elle reflète parfaitement les grands traits tectoniques de la région. Cette carte montre clairement que le secteur étudié est subdivisé en deux domaines distincts. Le premier septentrional fortement structuré et le second méridional peu déformé. La limite entre ces deux domaines peut être matérialisée par le front de chevauchement, limitant au Nord, la dépression centrale du bassin d'Ouarzazate. Le fort contraste des caractéristiques structurales entre les deux domaines apparait nettement sur les sections sismiques transversales (fig. 5 et 6 ). La figure 5 montre quelques aspects de la déformation intense qui caractérise le domaine septentrional. Cependant, le caractère tectonique relativement calme du domaine méridional est clairement illustré par la figure 6 .

La figure 5 montre que le domaine septentrional est caractérisé par une structuration intense qui se manifeste par des plis, des plis-chevauchants et des écailles. Ces structures, de vergence Sud, se suivent et se relaient d'Est en Ouest. Elles sont orientées en direction E-W à ENE-WSW c.-à-d. parallèlement aux structures locales du Haut-Atlas. L'examen des données sismiques montrent clairement que cette structuration est liée à l'orogenèse atlasique d'âge oligocène. En effet, plusieurs sections révèlent que la déformation affecte clairement la série stratigraphique jusqu'au Paléocène et épargne les formations néogènes. Des chevauchements à vergence nord d'extension limitée ont également pu être identifiés. Ces accidents de faible extension peuvent être qualifiés de «back thrust fault" (fig. 5) qui cor- 


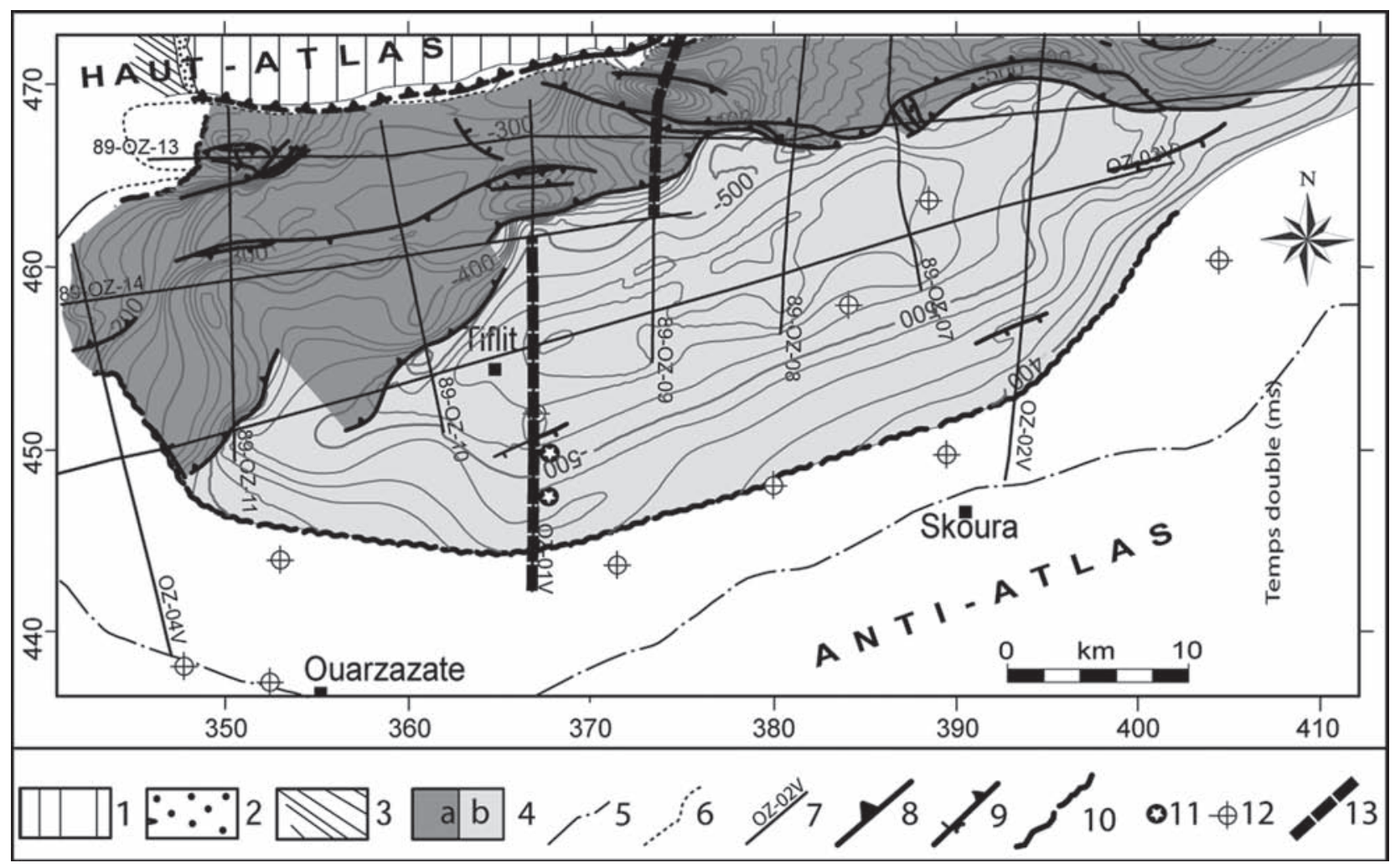

Fig. 4.-Carte des isochrones de la base de l'Eocène (bassin d'Ouarzazate). 1. Paléozoïque de la boutonnière de Skoura. 2. Trias autochtone. 3. Crétacé autochtone. 4. a) Domaine septentrional, b) Domaine méridional. 5. Limite septentrionale des affleurements du Précambrien de l'Anti-Atlas. 6. Limite sud des affleurements subautochtones du Crétacé et du Paléogène de la zone sud atlasique. 7. Profil sismique. 8. Faille inverse. 9. Faille normale. 10. Limite méridionale de la base de l'Eocène. 11. Forage pétrolier. 12. Forage hydrogéologique. 13. Emplacements des figures 5 et 6.

respondent à des failles d'accommodation ou de réajustement en liaison avec la mise en place des structures principales à vergence sud. Par ailleurs, la carte d'isochrone révèle l'existence sur la bordure sud de la boutonnière de Skoura, d'une importante faille inverse chevauchante vers le Sud. Celle-ci serait responsable du soulèvement de cette boutonnière et de sa mise en place. Plus au Sud, en direction de l'avant-pays anti-atlasique, on passe brusquement au domaine méridional qui est structuralement plus calme et où la tectonique atlasique est relayée par une déformation souple. Cette dernière est soulignée par l'existence d'une vaste dépression synclinale de direction ENE-WSW qui occupe le centre du bassin d'Ouarzazate et qui se poursuit au Sud par une importante structure monoclinale à faible pendage vers le Nord (fig. 6).

D'un point de vue hydrogéologique, la caractérisation de la géométrie 3D de la base de l'Éocène permet de se rendre compte clairement des variations latérales de la puissance de ce système aquifè- re. En effet, compte tenu du fait que le toit de cet étage correspond à une surface plus ou moins plane qui représente la discordance du Mio-Pliocène sur l'Éocène, les ondulations observées sur la carte d'isochrones (fig. 7) reflèteraient des variations de l'épaisseur de l'Éocène. De telles variations se traduisent par l'existence de plusieurs dépressions qui constituent des zones potentielles de convergence et d'accumulation des eaux souterraines, et par conséquent des zones potentiellement productives (ZPP) compte tenu (i) de la nature perméable de la majorité des formations constituant cet étage (calcaires, marnes, grès rouges et conglomérats) et (ii) de la forte puissance de ces formations au sein des dépressions. On ne dispose pas d'informations sur les étendus réelles de ces cuvettes, c'est pourquoi, on s'est contenté d'en tracer des limites indicatives. Parmi les zones potentiellement productives mises en évidence, les plus importantes sont représentées sur la figure 7. La première baptisée ZPP1 correspond à la grande cuvette synclinale décrite précé- 


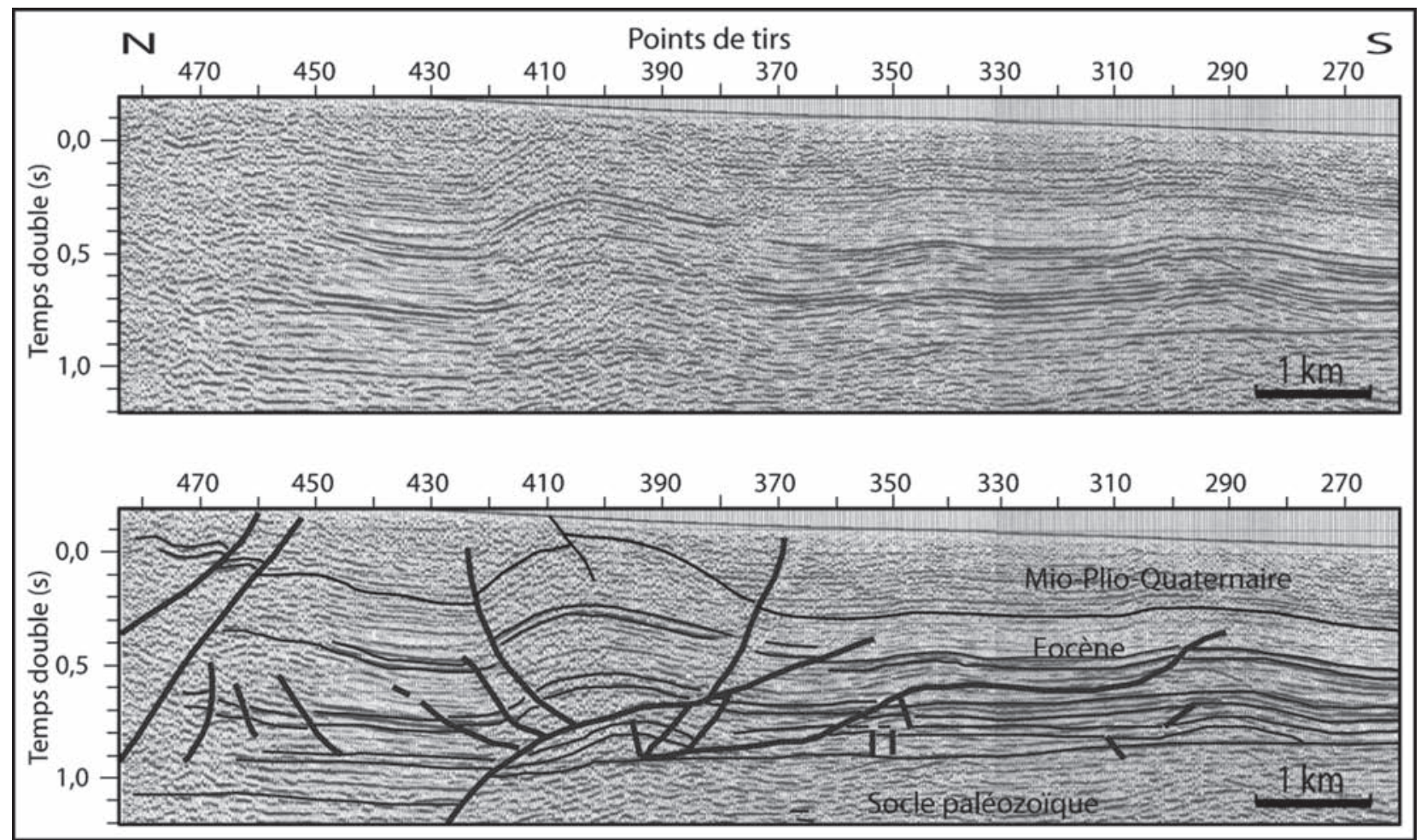

Fig. 5.-Section sismique montrant quelques aspects de la déformation intense affectant le domaine septentrional (Partie nord du profil 89-OZ-09).

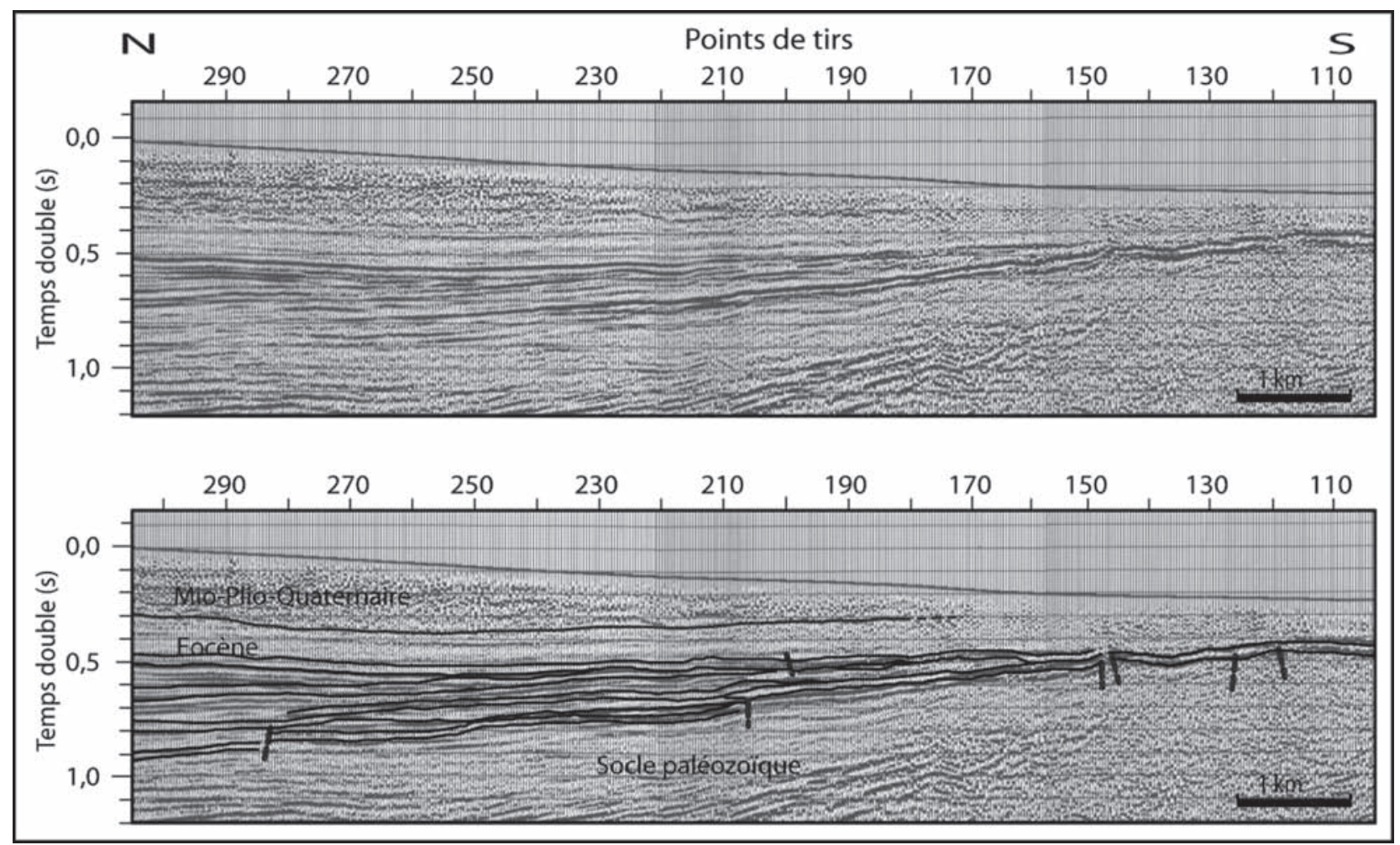

Fig. 6.-Section sismique illustrant le caractère peu déformé du flanc sud du bassin d'Ouarzazate (Partie sud du profil OZ-O1V). 


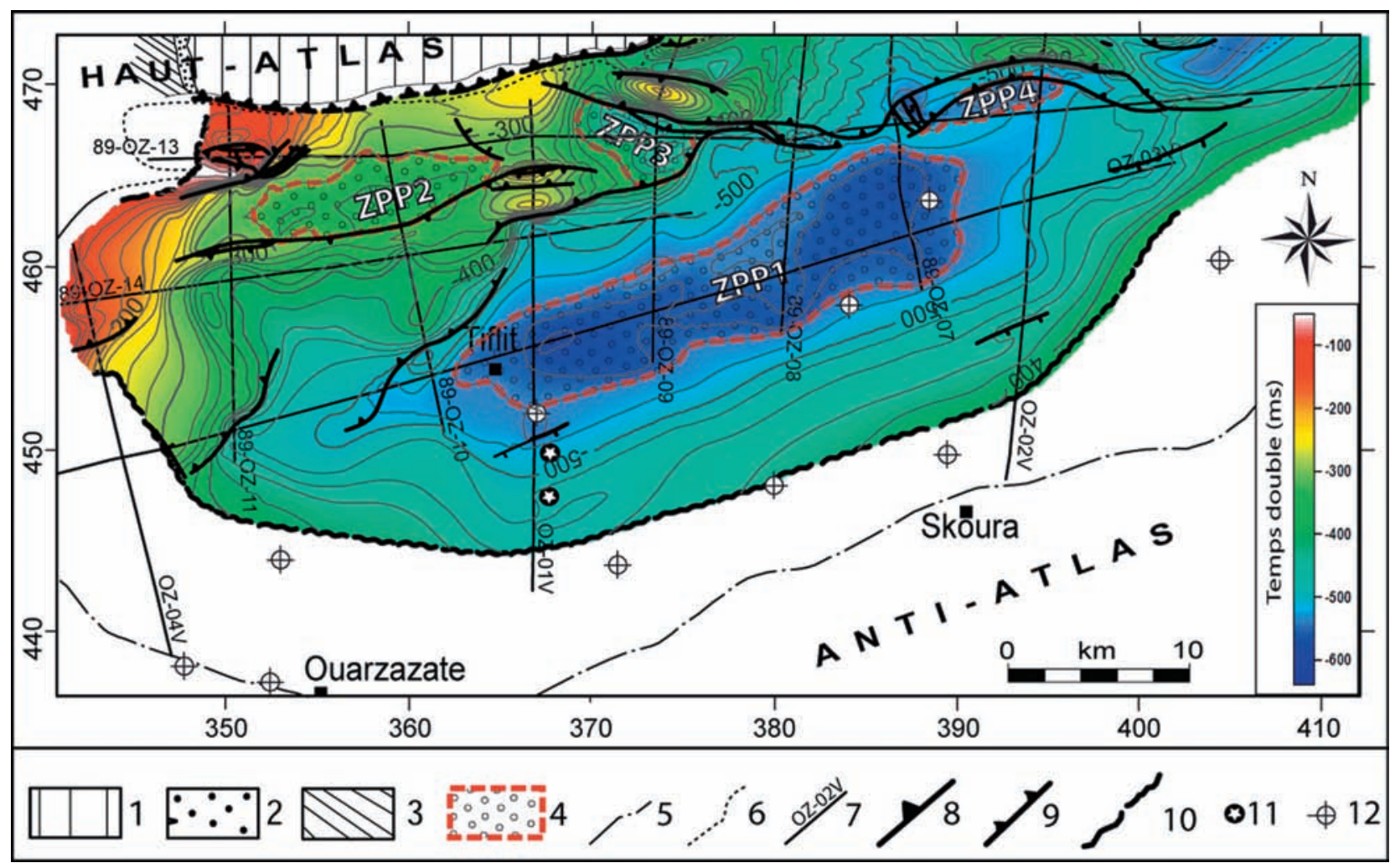

Fig. 7.-Carte des isochrones de la base de l'Eocène (bassin d'Ouarzazate). 1. Paléozoïque de la boutonnière de Skoura. 2. Trias autochtone. 3. Crétacé autochtone. 4. Zone potentiellement productives (ZPP). 5. Limite septentrionale des affleurements du Précambrien de l'Anti-Atlas. 6. Limite sud des affleurements subautochtones du Crétacé et du Paléogène de la zone sud atlasique. 7. Profil sismique. 8. Faille inverse. 9. Faille normale. 10. Limite méridionale de la base de l'Eocène. 11. Forage pétrolier. 12. Forage hydrogéologique.

demment qui occupe le centre du bassin d'Ouarzazate. Les autres d'étendue plus restreinte, formant un alignement est-ouest, se situent au Nord de la première. Les forages visant l'exploitation des eaux souterraines du système aquifère éocène devraient prioritairement cibler le cœur de ces zones.

La carte d'isochrones de la base de l'Éocène qui s'apparente à un schéma structural de la zone d'étude met clairement en évidence le rôle de la tectonique dans l'individualisation de ces cuvettes qui apparaissent toutes partiellement bordées par des accidents. Cela nous amène à considérer que le système aquifère éocène est un système compartimenté. En outre, les multiples failles qui hachent ce système aquifère ont d'importantes implications hydrogéologiques car celles-ci constituent des drains qui influent énormément sur l'écoulement des eaux souterraines en assurant notamment une certaine connectivité entre les différents aquifères ou les différentes couches perméables d'un même aquifère.

\section{REMERCIEMENTS}

Les auteurs remercient les membres de la direction d'exploration de l'Office National des Hydrocarbures et des Mines (ONHYM) pour leur collaboration et pour les documents mis à notre disposition.

Ce travail a été réalisé, en partie, dans le cadre d'un projet de coopération OTAN entre l'Université Cadi Ayyad et l'Université Autonome de Barcelone (Réf.: EST.CLG.980144). Nous remercions vivement nos partenaires espagnols et spécialement nos collègues Monsieur A. Teixell et madame M.L. Arboleya.

\section{Références}

Agoussine, M.; Saidi, M.E. \& Igmoullan, B. (2004). Reconnaissance des ressources en eau du bassin d'Ouarzazate (Sud-Est marocain). Bulletin de l'Institut Scientifique, Rabat, section Sciences de la Terre, 26: 81-92.

Amrhar, M. (2002). Paléocontraintes et déformations synet post-collision Afrique-Europe identifiées dans la couverture mésozoïque et cénozoïque du Haut Atlas occidental (Maroc) Syn- and post-collision Africa-Europe palaeostresses and deformations identified in the West 
High-Atlas Mesozoic and Cenozoic cover (Morocco). Comptes Rendus Geosciences, 334: 279-285

Cappetta, H.; Jaeger, J.J.; Sabatier, B.; Sigé, B.; Sudre, J. \& Vianey-Liaud, M. (1987). Complément et précisions biostratigraphiques sur la faune paléocène à Mammifères et Sélaciens du bassin d'Ouarzazate (Maroc). Tertiary Research, 8: 625-648.

Costagliola, A. \& Belkhir, M. (1992). Synthèse géologique, géophysique et pétrolière du bassin d'Ouarzazate et du bassin d'Errachidia Occidental. Rapport interne ONAREP.

Dutour, A. \& Ferrandini, J. (1985). Nouvelles observations néotectoniques dans le Haut-Atlas de Marrakech et le Haouz central (Maroc): apports sur l'évolution récente d'un segment du bâti atlasique. Revue de Géologie Dynamique et de Géographie Physique. 26: 285-297.

El Harfi, A.; Lang, J. \& Salomon, J.; (1996). Le remplissage continental cénozoïque du bassin d'avant-pays d'Ouarzazate: implications sur l'évolution géodynamique du Haut Atlas central (Maroc). Comptes Rendus de l'Académie des Sciences Série II, 323: 623-630.

Fraissinet, C.E.; Zouine, M.; Morel, J.-L.; Poisson, A.; Andrieux, J. \& Faure-Muret, A. (1988). Structural evolution of the southern and northern central High Atlas. In: The Atlas System of Morocco (Jacobshagen, V., Ed.), Springer-Verlag, Berlin, 273-291.

Frizon de Lamotte, D.; Saint Bézar, B.; Bracène, R. \& Mercier, E. (2000). The two main steps of the Atlas building and geodynamics of the western Mediterranean. Tectonics, 19: 740-761.

Görler, K.; Helmdach, F.-F.; Gaemers, P.; Heissig, K.; Hinsch, W.; Mèdler, K.; Schwarzhans, W. \& Zucht, M. (1988). The uplift of the Central Atlas as deduced from Neogene continental sediments of the Ouarzazate province, Morocco. In: The Atlas System of Morocco (Jacobshagen, V., Ed.), Springer-Verlag, Berlin, 361-404.
Jaffal, M., Kchikach, A., Lefort, J.P. \& Hanich, L. (2002). Contribution à l'étude d'une partie du bassin d'Essaouira (Maroc) par sismique réflexion, Comptes Rendus Geoscience 334: 229-234

Jossen, J.A. \& Filali Moutei, J. (1988). Bassin d'Ouarzazate, synthèse stratigraphique et structurale. Contribution à l'étude des aquifères profonds - Projet PNUD DRPE (Direction de la Recherche et de la planification de l'Eau) MOR /86/004- Exploration des eaux profondes. Rapport Inédit, $38 \mathrm{p}$.

Laville, E.; Lesage, J.L. \& Seguret, M. (1977). Géométrie, cinématique (dynamique) de la tectonique atlasique sur le versant sud du Haut Atlas marocain, aperçu sur la tectonique hercynienne et tardi-hercynienne. Bulletin de la Société Géologique de France, 7: 527-539.

Morel, J.-L., Zouine, M. \& Poisson, A., (1993). Relations entre la subsidence des bassins moulouyens et la création des reliefs atlasiques (Maroc): Un exemple d'inversion tectonique depuis le Néogène. Bulletin de la Société Géologique de France, 164: 79-91.

Morel, J.-L.; Zouine, M.; Andrieux, J. \& Faure-Muret, A. (2000). Déformations néogènes et quaternaires de la bordure nord haut-atlasique (Maroc): rôle du socle et conséquences structurales. Journal of African Earth Sciences 30: 119-131.

Sébrier, M.; Siame, L.; Zouine, E.M.; Winter, T.; Missenard, Y. \& Leturmy, P. (2006). Active tectonics in the Moroccan High Atlas. Comptes Rendus Geosciences, 338: 65-79

Wessel, P. \& Smith, W. H. F. (1995). A new version of the generic mapping tool (gmt), EOS, Transactions, American Geophysical Union 76: 329.

Recibido el 20 de febrero de 2009

Aceptado el 8 de junio de 2009

Publicado online el 15 de diciembre de 2009 